\title{
CR Resarch Square

\section{Prognostic death factors in SHLH children with MODS receiving CRRT: a multicenter prospective nested case-control study}

\section{Yun Cui}

Shanghai Children's Hospital

Jingyi Shi

Shanghai Children's Hospital

Yijun Shan

Shanghai Children's Hospital

Chunxia Wang

Shanghai Children's Hospital

\section{Yuqian Ren}

Shanghai Children's Hospital

\section{Guoping Lu}

Children's Hospital of Fudan University

\section{Gangfeng Yan}

Children's Hospital of Fudan University

\section{Xiaodong Zhu}

Shanghai Jiaotong University School of Medicine Xinhua Hospital

\section{Yueniu Zhu}

Shanghai Jiaotong University School of Medicine Xinhua Hospital

\section{Ying Wang}

Shanghai Children's medical center

\section{Hong Ren}

Shanghai Children's medical center

\section{Yucai Zhang ( $\nabla$ zyucai2018@163.com )}

Shanghai Children's Hospital https://orcid.org/0000-0002-4905-3600

\section{Research}

Keywords: outcomes, mortality, Continuous renal replacement therapy, secondary hemophagocytic lymphohistiocytosis, pediatric intensive care unit

Posted Date: February 11th, 2020 
DOI: https://doi.org/10.21203/rs.2.23182/v1

License: (c) (1) This work is licensed under a Creative Commons Attribution 4.0 International License. Read Full License 


\section{Abstract}

Background: Multiple organ dysfunction syndrome (MODS) with secondary hemophagocytic lymphohistiocytosis (SHLH) causes significant mortality, while continuous renal replacement therapy (CRRT) is commonly conducted. The objective is to identify the predictor factors associated with poor outcomes in pediatric patients with SHLH -associated MODS who received CRRT.

Methods: A multicenter prospective nested case-control study in four PICUs of tertiary university children's hospital in Shanghai from September 2013 to August 2018. We prospectively studied 52 SHLHassociated MODS pediatric patients receiving CRRT.

Results: Overall PICU mortality rate was $46.15 \%(24 / 52)$. Less respiratory $(28.6 \%$ vs. $87.5 \%, P<0.001)$ or cardiovascular dysfunction ( $25 \%$ vs. $83.3 \%, \mathrm{P}<0.001)$ caused in survivors at CRRT initiation, as well as reduced demands of mechanical ventilation and vasoactive agents ( $28.6 \%$ vs. $87.5 \%, 17.9 \%$ vs. $66.7 \%$, both $P$ <0.001). Non-survivors had higher levels of serum lactate dehydrogenase $(1404.5(713.25,2793)$ vs. $982.7(692,1461)(\mathrm{U} / \mathrm{L}), \mathrm{P}=0.037)$, lactic acid $(1.9(1.3,4.53)(\mathrm{mmol} / \mathrm{L})$ vs. $1.65(0.8,2.45), \mathrm{P}=0.034)$, triglyceride $(2.88(1.94,5.08)(\mathrm{mmol} / \mathrm{L})$ vs. $2.41(1.63,3.32), \mathrm{P}=0.032)$ and IL-6 $(28.66(17.77,113.63)$ $(\mathrm{pg} / \mathrm{ml})$ vs.0.98 $(0.1,4.63) \mathrm{P}=0.000)$. More than 3 organ dysfunction (Odd ratio [ OR ] : $3.464 ; 95 \%$ confidence interval [ $\mathrm{Cl}$ ] [1.018-11.788], $\mathrm{P}=0.047$ ), and the serum IL-6 level higher than $13.12 \mathrm{pg} / \mathrm{mL}$ ( OR $: 1.388 ; 95 \% \mathrm{Cl}$ [1.058-1.821], $\mathrm{P}=0.018$ ) were two independent risk factors for mortality.

Conclusions: The number of organ dysfunction and IL-6 levels at CRRT initiation are the independent risk factors for mortality in SHLH-associated MODS patients.

\section{Background}

Secondary hemophagocytic lymphohistiocytosis (SHLH) without familial history or known genetic predisposition is considered an overwhelming and life-threatening systemic inflammatory syndrome. SHLH can be related to infectious diseases (IAHS), associated with autoimmune diseases (MAS), or due to malignancies $(\mathrm{M}-\mathrm{HLH})$. Over half of all the SHLH cases culminate in multiple organ failure, with up to $86 \%$ requiring admission to the intensive care unit (ICU). Among ICU patients, SHLH are usually triggered by infection disease and sepsis[1].

Appling initial chemotherapy including etoposide combined with steroids and cyclosporine A in a timely fashion is important for survival. However, despite advances and improvements in chemotherapy, in $20 \%$ of cases, SHLH patients do not respond to conventional treatment, many of these patients die of rapid deterioration due to severe sepsis and MODS[2]. The mortality rate in SHLH with MODS patients has remained unacceptably high, at greater than $50 \%$ in some studies[3]. In children, SHLH may present with more acute fulminant manifestations and the incidence of MODS was higher than adults[4,5]. Poor outcomes in children with SHLH-associated MODS have led to a call for action to improve early diagnosis, institute new preventive measures, and develop new treatments to improve clinical outcomes. 
Recently, Continuous renal replacement therapy (CRRT) has evolved from standard renal replacement therapy into an especially unique role in "mutiple-organ support technology" for MODS patients. CRRT is very effective in removal of inflammatory mediators, as well as maintaining fluid balance and hemodynamic stability for critical ill patients[6]. Aggressive application of CRRT was found to decrease mortality in adult patients with septic shock and MODS[7]. SHLH patients may need CRRT for renal replacement, inflammatory mediator or cytokine removal. Similarly, CRRT is an important treatment for severe SHLH patients, although there are insufficient studies regarding the efficacy and indications for CRRT in the pediatric HLH ICU population. We previously found that high-volume hemofiltration may improve organ function by decreasing tumor necrosis factor-a and interleukin-6, which might be an effective adjunctive treatment in secondary hemophagocytic lymphohistiocytosis[8]. The mortality among children with MODS requiring CRRT continues to be very high. Therefore, it is important for physicians to identify patients in whom aggressive treatment may offer recovery or those who may benefit from CRRT.

To the best of our knowledge, there are no available data on outcomes of critically ill children with SHLH and MODS receiving CRRT and identifying relevant prognostic factors. In this study, we initially analyzed survival outcomes among 52 pediatric patients with SHLH and MODS receiving CRRT. Then we assessed predictive factors for survival outcome. PRISM scores were used to assess whether the observed effect was independent of severity of illness.

\section{Methods}

\section{Design and setting}

We performed a prospective multicentre observational cohort study between August 2013 and July 2018 from 4 tertiary care PICUs in Shanghai. Fifty-two critically ill children were enrolled in this study. The protocol was approved by each institution's ethics/investigation review board (2016R011-F01).Informed to participation in the study consent was obtained from the guardians of the patients.

Eligibility criteria for this study were as follows: 1) aged between 1 month and 18 years, 2) meet HLH2004 and MODS diagnostic criteria, 3) treated with HLH-2004 protocol, 4) receiving CRRT during the PICU stay[9]. MODS was defined as the underlying primary disease process leading to at least two organ system dysfuction at any time during PICU stay[10]. Patients with familial history or known genetic predisposition(primary HLH) were excluded from analysis. Data from enrolled patients were entered into standardized electronic case report forms (CRF).

\section{CRRT treatment}

The indications for CRRT in our study were as follow: 1 ) hypercreatinemia or azotemia (creatinine levels of $>2 \mathrm{mg} / \mathrm{dL}$; 2) blood urea nitrogen levels of $>40 \mathrm{mg} / \mathrm{dL}$ ); 3) oliguria (urine output of $<0.5 \mathrm{~mL} / \mathrm{kg} / \mathrm{h}$ ); 4) fluid overload $>10 \%$; 5) acute liver failure complicated with hepatorenal syndrome or hyperammonaemia; 
6) severe electrolyte imbalance which did not respond immediately to conventional therapy (hyperkalaemia $>7 \mathrm{mmol} / \mathrm{L}$; hypernatraemia $>160 \mathrm{mmol} / \mathrm{L}$ or hyponatraemia $<110 \mathrm{mmol} / \mathrm{L}$ ).

Vascular access was obtained with 5F dual-lumen ( Arrow, Teleflex Inc, Limerick, PA, USA), 6.5F-12F central venous catheters (GamCath; Gambro, Colombes, France) in the right internal jugular or femoral vein, according to patient body weight.

Two CRRT devices were used, including the Plasauto iQ21 (Asahi KASE, Japan), and PRISMA flex (Gambro Renal Products, France). Polyacrylonitrile AN69 or polysulfone hollow-fibre haemofilters were used, depending on the body surface area of the patient and on the pump employed. M10 filters (Gambro Renal Products, France) or AEF-03(Asahi KASE, Japan) were used in children weighing less than $5 \mathrm{~kg}$; M60 (Gambro Renal Products, France) or (Asahi KASE, Japan) was used in patients weighing between 5 and $35 \mathrm{~kg}$, and M100 (Gambro Renal Products, France) or AEF-10 (Asahi KASE, Japan) in children weighing over $35 \mathrm{~kg}$. Thirty-three patients $(63.5 \%)$ received $\mathrm{CVVH}$, and nineteen patients $(36.5 \%)$ received continuous venovenous hemodiafiltration (CVVHDF) with an ultrafiltrate flow rate of $35-50 \mathrm{~mL} / \mathrm{kg} / \mathrm{hr}$. Hemofilters changes were scheduled every $24 \mathrm{~h}$ or when clotted.

The dosage of was 5-20U/kg.h to maintain activated partial thromboplastin time (APTT) with 1.5-2 folds of normality during CRRT. Anticoagulation was achieved with heparin adjusted to a target activated partial thromboplastin time (APTT) of 41.5- to 2 -fold of normal value. The regional citrate anticoagulation (RCA) procedure was conducted according to the guidelines from the Prismaflex operator's manual. When sodium citrate was used as anticoagulation, the target post-filter ionized calcium level of $0.25-$ $0.35 \mathrm{mmol} / \mathrm{L}$ was maintained. The citrate effect was neutralized using a continuous calcium infusion of $10 \%$ calcium gluconate to maintain ionized calcium blood levels between 1.0 and $1.2 \mathrm{mmol} / \mathrm{L}$.

\section{Data collect}

Data on patients' demographics and laboratory investigations during the first 3 days of PICU admission were prospectively collected by the attending intensivists according to standard practice in each PICU. In addition, during PICU stay, data regarding onset, duration and type of organ dysfunction, Pediatric Risk of Mortality III (PRISM III) scores were registered by the same intesivist daily. The following data were also recorded: age, sex, reasons for PICU admission, primary disease, comorbidities and family history of HLH.

Initiation of CRRT and prescription of replacement and dialysis doses were based on the decisions of the PICU attending intensivist. At the time of starting CRRT, the following data were gathered prospectively: reasons for CRRT initiation, days in PICU until CRRT initiation, total fluid intake and output from admission to the PICU until initiation of CRRT, mechanical ventilation and the number of vasoactive drugs to support respiratory and circulation.

CRRT is performed either as continuous venovenous hemofiltration (CVVH) or haemodiafiltration (CVVHDF). During CRRT therapy, a daily record was kept of maximum dose of heparin, ultrafiltration rate, life of each filter, CRRT-related complications and PICU mortality. Reasons to terminate CRRT were as follows: patient death/withdrawal of support, inability to continue CRRT, or regained organ function. 
Laboratory values were recorded upon admission to the hospital, admission to the PICU, and initiation of CRRT.

\section{Statistical Methods}

The statistical analyses were performed with IBM SPSS Statistics V.22.0 (SPSS Inc, Chicago). Continuous variables were summarized as means \pm SDs for normal distribution data and as median (interquartile range) for abnormal distribution data. Independent-samples t test (for normal distribution data), MannWhitney U tests (for abnormal distribution data), or Chi-square (for categorical variables) was used to compare parameters in the two groups. Proportions were compared using the $\chi 2$ test or by linear-by linear $\chi 2$ association test in case of ordinal categorical variables. Univariate and multivariate analyses were performed to analyze the influence of each factor on PICU mortality. The ability of factors to predict hospital mortality was tested using receiver operating characteristic (ROC) curve performed using STATA 15.0 MP (College Station, Texas, USA). Cut-off values were identified using the highest Youden index $\left(\right.$ sensitivity + specificity $\left.^{-1}\right)[11]$. Significance was taken as a $P$ value less than 0.05 .

\section{Result}

\section{Patient's characteristics}

\section{Baseline Characteristics of Pediatric Patients With SHLH}

Over the 5-year study period, a total of 114 patients were screened and 52 cases were enrolled. (Fig. 1). Among these patients, the mean age was 24.5 months $(12-89 \mathrm{mo})$ and 28 patients $(53.8 \%)$ were male. Of 52 patients with SHLH required CRRT, 24 patients died and overall hospital mortality was $46.15 \%$. The characteristics of the patients included are shown in Table 1The etiology of the SHLH disease was most frequently infection-related $(n=46,88.5 \%)$, among which $87.0 \%$ was EBV-SHLH $(n=40)$. The common indications for PICU admission was hypoxemia $(34.6 \%, n=18)$, cardiovascular dysfunction $(28.9 \%, n=$ $15)$, recurrent seizures $(18.9 \%, n=10)$ or AKI $(17.3 \%, n=9)$. The main reasons for CRRT were fluid overload (FO) $(42.3 \%, n=22)$, hepatic dysfunction $(30.8 \%, n=16)$, acute kidney injury (AKI) $(17.3 \%, n=9)$, and severe electrolyte imbalance $(7.5 \%, n=4)$. The femoral vein was catheterized in $69.2 \%$ of patients (36/52), and the jugular vein in $30.8 \%(16 / 52)$. At the time of starting CRRT, mechanical ventilation required in $44.1 \%$ (33/52) patients, vasoactive drugs continuously infused in $28.8 \%$ (14/52) patients, and $23.1 \%(12 / 52)$ of them received diuretics. Anticoagulation was achieved using heparin in $69 \%$ of patients $(36 / 52)$ and citrate in $31 \%$ of patients $(16 / 52)$. The mean length of CRRT was $43(24,86.5)$ hours.

\section{Risk factors for PICU mortality}

Overall PICU mortality for all admissions was $46.2 \%$ (24/52). There were no significant difference between survivors and nonsurvivors with respect to age, sex, EBV infection, Pediatric Risk of Mortality III (PRISM III) score at PICU admission, and the type of SHLH. For all patients, the factors that were 
significantly different between survivors and nonsurvivors were the length of PICU stay, number of organ dysfunction, mechanical ventilation, and vasoactive agents needed.

Table 2 lists data comparing laboratory variables for survivors versus non-survivors. TBIL, DBIL, ALT, AST, ALB, PT, FIB, BUN and CR, as well as WBC, HB, PLT level at CRRT initiation were no different for survivors compared to non-survivors. In contrast, non-survivors had significantly higher levels of serum LDH $(1404.5(713.25,2793)$ vs. $982.7(692,1461)(\mathrm{U} / \mathrm{L}), \mathrm{P}=0.037), \operatorname{LAC}(1.9(1.3,4.53)(\mathrm{mmol} / \mathrm{L})$ vs. $1.65(0.8$, 2.45), $\mathrm{P}=0.034)$, triglyceride $(2.88(1.94,5.08)(\mathrm{mmol} / \mathrm{L})$ vs. $2.41(1.63,3.32), \mathrm{P}=0.032)$ at CRRT initiation. 
Table 1

Demographic and clinical data at CRRT initiation

\begin{tabular}{|c|c|c|c|c|}
\hline & $\begin{array}{l}\text { ALL } \\
(n=52)\end{array}$ & $\begin{array}{l}\text { Survivor } \\
(n=28)\end{array}$ & $\begin{array}{l}\text { Non- } \\
\text { survivor } \\
(n=24)\end{array}$ & $\mathbf{p}$ \\
\hline Male, n (\%) & $28(53.8)$ & $18(64.3)$ & $10(41.7)$ & 0.103 \\
\hline Age,month,median (IQR) & $\begin{array}{l}24.5(12.0, \\
89.0)\end{array}$ & $\begin{array}{l}22.0(13.5, \\
89.0)\end{array}$ & $\begin{array}{l}26.5(11.5 \\
75.5)\end{array}$ & 0.985 \\
\hline Length of PICU stay, day, median (IQR) & $\begin{array}{l}24.0(14.0 \\
33.0)\end{array}$ & $\begin{array}{l}30.5(22.5 \\
40.5)\end{array}$ & $\begin{array}{l}15.0(6.0 \\
23.0)\end{array}$ & 0.000 \\
\hline Expenses of PICU RMB, median (IQR) & $\begin{array}{l}83810 \\
(54678, \\
16047)\end{array}$ & $\begin{array}{l}88946.35 \\
(59844, \\
122177)\end{array}$ & $\begin{array}{l}78759 \\
(45493 \\
130566)\end{array}$ & 0.941 \\
\hline PRISM score, median (IQR) & $15(13,21)$ & $14(11,21)$ & $17(12,23)$ & 0.427 \\
\hline \multicolumn{5}{|l|}{ Type of SHLH } \\
\hline IAHS, n (\%) & $46(88.5)$ & 23(82.1) & 23(95.8) & 0.123 \\
\hline EBV-SHLH, n (\%) & $40(76.9)$ & 19(67.9) & $21(87.5)$ & 0.094 \\
\hline MAS, n (\%) & $6(11.5)$ & $5(17.9)$ & $1(4.2)$ & 0.123 \\
\hline \multicolumn{5}{|l|}{ Organ dysfunction at CRRT, $\mathrm{n}(\%)$} \\
\hline Respiratory & $29(55.8)$ & $8(28.6)$ & $21(87.5)$ & $\begin{array}{l}<.001 \\
0.001\end{array}$ \\
\hline Cardiovascular & $27(51.9)$ & $7(25.0)$ & $20(83.3)$ & $\begin{array}{l}< \\
0.001\end{array}$ \\
\hline Gastrointestinal & $20(38.5)$ & $3(10.7)$ & 17(70.8) & $\begin{array}{l}< \\
0.001\end{array}$ \\
\hline Hepatic & $45(86.5)$ & $24(85.7)$ & $21(87.5)$ & 0.851 \\
\hline Hematologic & $48(92.3)$ & $26(92.9)$ & 22(91.7) & 0.872 \\
\hline Renal & $16(30.8)$ & $7(25.0)$ & $9(37.5)$ & 0.330 \\
\hline Neurologic & $16(30.8)$ & $7(25.0)$ & $9(37.5)$ & 0.330 \\
\hline Number of Organ dysfunction, median (IQR) & $\begin{array}{l}\text { 4.0(2.5, } \\
5.0)\end{array}$ & $\begin{array}{l}3.0(2.0 \\
3.5)\end{array}$ & $\begin{array}{l}5.0(5.0, \\
5.0)\end{array}$ & 0.000 \\
\hline Mechanical ventilation, $\mathrm{n}(\%)$ & $23(55.8)$ & $8(28.6)$ & 15(87.5) & 0.000 \\
\hline
\end{tabular}




\begin{tabular}{|lllll|}
\hline & $\begin{array}{l}\text { ALL } \\
(\mathbf{n = 5 2})\end{array}$ & $\begin{array}{l}\text { Survivor } \\
(\mathbf{n = 2 8})\end{array}$ & $\begin{array}{l}\text { Non- } \\
\text { survivor } \\
(\mathbf{n = 2 4 )}\end{array}$ & p \\
\hline $\begin{array}{l}\text { vasoactive agents, } \\
\mathrm{n}(\%)\end{array}$ & $21(40.4)$ & $5(17.9)$ & $16(66.7)$ & 0.000 \\
\hline Indication for CRRT, $\mathrm{n}(\%)$ & & & & \\
\hline AKI & $12(22.2)$ & $6(21.4)$ & $6(25.0)$ & 0.761 \\
\hline FO & $9(17.3)$ & $5(17.9)$ & $4(16.7)$ & 0.910 \\
\hline $\begin{array}{l}\text { Remove } \\
\text { Inflammatory }\end{array}$ & $52(100)$ & $28(100)$ & $24(100)$ & 1.000 \\
\hline Mediators & & & & \\
\hline CRRT mode, $n$ (\%) & & & & 0.359 \\
\hline CVVH & $14(26.9)$ & $9(32.1)$ & $5(20.8)$ & 0.359 \\
\hline $\begin{array}{l}\text { Interval time of CRRT initiation after SHLH } \\
\text { diagnosis } \geq 3 \text { days, } n(\%)\end{array}$ & $38(73.1)$ & $19(67.9)$ & $19(79.2)$ & 0.275 \\
\hline \begin{tabular}{l} 
mean length of CRRT (h), median (IQR) \\
\hline
\end{tabular} & $51(98.1)$ & $28(100)$ & $23(95.8)$ & \\
\hline
\end{tabular}


Table 2

The laboratory variables for survivors versus nonsurvivors.

\begin{tabular}{|c|c|c|c|c|}
\hline $\begin{array}{l}\text { Biological } \\
\text { Parameters } \\
\text { median (IQR) }\end{array}$ & $\begin{array}{l}\text { ALL } \\
n=52\end{array}$ & $\begin{array}{l}\text { Survivor } \\
n=28\end{array}$ & Nonsurvivor $\mathrm{n}=24$ & $\mathbf{p}$ \\
\hline TBIL, umol/L & $25.18(8.09,56.73)$ & $15.53(5.76,51.76)$ & $44.02(11.47,72.89)$ & 0.128 \\
\hline DBIL, umol/L & $13.99(4.08,40.66)$ & $8.66(3.03,28.03)$ & $34.37(5.23,43.84)$ & 0.061 \\
\hline ALT, umol/L & $\begin{array}{l}\text { 121.5(68.96, } \\
256.06)\end{array}$ & $147.0(74.25,256.18)$ & $83.96(39.18,318.78)$ & 0.313 \\
\hline ALB,$g / L$ & $28.39(24.89,32.74)$ & $29.42(25.05,33.75)$ & $26.75(24.75,32.69)$ & 0.245 \\
\hline LDH, IU/L & $\begin{array}{l}1030.5(720.25 \\
1868)\end{array}$ & $982.7(692,1461)$ & $1404.5(713.25,2793)$ & 0.037 \\
\hline $\mathrm{BUN}, \mathrm{mmol} / \mathrm{L}$ & $4.14(2.96,7.53)$ & $3.84(2.79,5.28)$ & $4.8(2.79,10.11)$ & 0.163 \\
\hline CR, umol/L & $24.77(18.45,41.8)$ & $23.38(18.15,35.75)$ & $26.68(17.78,81.08)$ & 0.409 \\
\hline PT, s & $14.6(13.75,18.6)$ & $14.2(12.45,18.23)$ & $16.85(14.03,20.2)$ & 0.057 \\
\hline FIB, g/L & $0.59(0.35,1.19)$ & $0.54(0.32,1.68)$ & $0.66(0.34,0.91)$ & 0.666 \\
\hline $\mathrm{LAC}, \mathrm{mmol} / \mathrm{L}$ & $1.7(1.15,2.93)$ & $1.65(0.8,2.45)$ & $1.9(1.3,4.53)$ & $0.034^{*}$ \\
\hline WBC, $10^{9} / \mathrm{L}$ & $3.30(1.29,7.01)$ & $3.62(1.45,7.11)$ & $2.94(1.02,8.22)$ & 0.441 \\
\hline Hemoglobin, g/L & $85(73,96.5)$ & $81.5(68.25,99)$ & $89(77.25,97.5)$ & 0.797 \\
\hline Platelets, $10^{9} / \mathrm{L}$ & $63(34.25,84.75)$ & $66(27,85.75)$ & $59(35.75,96.75)$ & 0.582 \\
\hline $\begin{array}{l}\text { Triglyceride, } \\
\mathrm{mmol} / \mathrm{L}\end{array}$ & $2.65(1.81,3.67)$ & $2.41(1.63,3.32)$ & $2.88(1.94,5.08)$ & 0.024 \\
\hline $\begin{array}{l}\text { Ferritin > } \\
1500 \mathrm{ng} / \mathrm{ml}, \mathrm{n} \\
(\%)\end{array}$ & $43(82.69)$ & $22(78.57)$ & $21(87.5)$ & 0.082 \\
\hline $\mathrm{IL}-6, \mathrm{pg} / \mathrm{mL}$ & $5.27(0.62,23.08)$ & $0.98(0.10,4.63)$ & $28.66(17.77,113.63)$ & 0.000 \\
\hline $\mathrm{IL}-1, \mathrm{pg} / \mathrm{mL}$ & $1.045(0.12,2.33)$ & $0.92(0.11,2.36)$ & $1.22(0.11,2.61)$ & 0.852 \\
\hline IL-10, pg/mL & $4.06(0.1,39.18)$ & $3.43(0.29,21.22)$ & $10.35(0.1,182.74)$ & 0.081 \\
\hline TNF-a, pg/mL & $1.45(0.10,10.45)$ & $1.44(0.10,10.31)$ & $1.45(0.1,12.75)$ & 0.383 \\
\hline
\end{tabular}

Total bilirubin (TBIL); Direct bilirubin (DBIL); Lactate dehydrogenase (LDH); Alanine transaminase (ALT); Blood Urea Nitrogen (BUN); Prothrombin Time (PT); fibrinogen (FIB); Lactic acid (LAC); white blood cell (WBC); interleukin (IL)

${ }^{*} \mathrm{p}<0.05$ 


\begin{tabular}{|lllll|}
\hline $\begin{array}{l}\text { Biological } \\
\text { Parameters } \\
\text { median (IQR) }\end{array}$ & $\begin{array}{l}\text { ALL } \\
\mathbf{n = 5 2}\end{array}$ & $\begin{array}{l}\text { Survivor } \\
\mathbf{n = 2 8}\end{array}$ & Nonsurvivor $\mathbf{n}=\mathbf{2 4}$ & $\mathbf{p}$ \\
\hline SCD25, pg/mL & $\begin{array}{l}30426.5(15950.46, \\
52028.6)\end{array}$ & $\begin{array}{l}29074.39(12526.45, \\
56262.46)\end{array}$ & $\begin{array}{l}36862.5(21893.18, \\
47356.85)\end{array}$ & 1.000 \\
\hline IL-8, pg/mL & $3.72(0.10,32.39)$ & $3.72(0.10-34.68)$ & $4.09(0.1,66.24)$ & 0.662 \\
\hline IL-12, pg/mL & $0.77(0.10,6.25)$ & $0.76(0.10,5.09)$ & $0.93(0.1,6.31)$ & 0.286 \\
\hline IL-18, pg/mL & $\begin{array}{l}344.15(95.73, \\
1626.42)\end{array}$ & $542.1(88.01,2259.71)$ & $326.16(98.96$, & 0.857 \\
\hline $\begin{array}{l}\text { Total bilirubin (TBIL); Direct bilirubin (DBIL); Lactate dehydrogenase (LDH); Alanine transaminase } \\
\text { (ALT); Blood Urea Nitrogen (BUN); Prothrombin Time (PT); fibrinogen (FIB); Lactic acid (LAC); white } \\
\text { blood cell (WBC); interleukin (IL) }\end{array}$ & & & \\
\hline *p<0.05 & & & & \\
\hline
\end{tabular}

Table 3

Predictive capacity for PICU mortality of the selected variables in patients with SHLH and MODS receiving CRRT.

\begin{tabular}{|llll|}
\hline Variables & Odds ratio & $95 \% \mathrm{Cl}$ & $\mathrm{P}$ \\
\hline IL-6 & 1.388 & $1.058-1.821$ & $0.018^{\star}$ \\
\hline Number of Organ dysfunction & 3.464 & $1.018-11.788$ & $0.047^{\star}$ \\
\hline LAC & 0.773 & $0.194-3.084$ & 0.715 \\
\hline Triglyceride & 1.633 & $0.814-3.279$ & 0.168 \\
\hline LDH & 1.732 & $0.528-3.252$ & 0.232 \\
\hline Age & 0.997 & $0.968-1.028$ & 0.863 \\
\hline Only factors with statistical significance in the univariate analyses were included, $* \mathrm{P}<0.05$ \\
\hline
\end{tabular}

In addition, patients with SHLH and MODS who died during their hospital displayed a significantly higher IL-6 $(28.66(17.77,113.63)(\mathrm{pg} / \mathrm{ml})$ vs.0.98 $(0.1,4.63) \mathrm{P}=0.000)$ at CRRT initiation when compared to those who survived. No difference was observed in serum IL-1, IL-10, TNF-a, SCD25, IL-8, IL-12 and IL-18 between patients discharged alive from PICU and those who died during PICU stay.

Logistic regression analysis using PICU mortality as the endpoint was performed on categorical and continuous variables. Regression modeling identified two independent risk factors of mortality: the number of Organ dysfunction (OR: 3.464; 95\% $\mathrm{Cl}$ [1.018-11.788], $\mathrm{P}=0.047$ ), and the serum IL-6 level (OR:1.388; 95\% Cl [1.058-1.821], $\mathrm{P}=0.018)$. Receiver Operating Characteristic (ROC) curve was used to evaluate the power of the two independent variables. The number of Organ dysfunction and the serum IL- 
6 level at PICU admission were found high discriminative power with AUC values respectively of 0.896 (SE 0.046, 95\% Cl 0.806-0.986) and 0.964 (SE 0.026, 95\% Cl 0.913-1.00).

Furthermore, we used the highest Youden Index to determine the most discriminative cut-off point for the number of Organ dysfunction and the serum IL- 6 level at PICU admission to predict PICU mortality (Table 4-5, Fig. 2). A value of serum IL-6 level of $13.12 \mathrm{pg} / \mathrm{ml}$ and the number of Organ dysfunction of 3 at CRRT initiation have been identified as cut-off points.

Table 4

ROC analysis of IL-6 and number of organ dysfunction in the PICU mortality of patients with SHLH and MODS

\begin{tabular}{|llllll|}
\hline Variables & $\begin{array}{l}\text { Cut-off } \\
\text { point }\end{array}$ & $\begin{array}{l}\text { Sensitivity } \\
\text { (\%) }\end{array}$ & $\begin{array}{l}\text { Specificity } \\
\text { (\%) }\end{array}$ & $\begin{array}{l}\text { positive } \\
\text { predictive value } \\
(\%)\end{array}$ & $\begin{array}{l}\text { negative } \\
\text { predictive value } \\
(\%)\end{array}$ \\
\hline $\begin{array}{l}\text { Number of Organ } \\
\text { dysfunction }\end{array}$ & 3.0 & 95.8 & 75.0 & $23 / 30(76.7)$ & $21 / 22(95.5)$ \\
\hline IL-6 & 13.12 & 91.7 & 100.0 & $22 / 22(100.0)$ & $28 / 30(93.3)$ \\
\hline $\begin{array}{l}\text { The highest Youden Index determined the most discriminative cut-off point for the number of Organ } \\
\text { dysfunction and the serum IL-6 level at PICU admission }\end{array}$ & \\
\hline
\end{tabular}

Table 5

ROC analysis of IL-6 and number of organ dysfunction in the PICU mortality of patients with SHLH and MODS

\begin{tabular}{|c|c|c|c|c|c|}
\hline \multirow[t]{2}{*}{ Variables } & \multirow[t]{2}{*}{ AUC } & \multirow[t]{2}{*}{ SE } & \multirow[t]{2}{*}{$\mathbf{P}$} & \multicolumn{2}{|c|}{$95 \%$ confidence interval } \\
\hline & & & & upper limit & lower limit \\
\hline Number of Organ dysfunction & 0.896 & 0.046 & $0.000 *$ & 0.806 & 0.986 \\
\hline IL-6 & 0.964 & 0.026 & $0.000 *$ & 0.913 & 1.000 \\
\hline${ }^{*} p<0.05$ & & & & & \\
\hline
\end{tabular}

\section{Discussion}

To our knowledge, our study is the largest, multicenter, prospective study of CRRT conducted in critically ill children with SHLH-associated MODS. Furthermore, we additionally reported the first data on detailing the PICU course and outcome of these patients. We observed that patients with SHLH and MODS received CRRT in PICU was associated with a high PICU mortality at $46.2 \%(22 / 52)$. Our data also demonstrated that the number of organ dysfunction and serum IL- 6 level at CRRT initiation higher than 3 and $13.12 \mathrm{pg} / \mathrm{ml}$ are independent risk factors of initial PICU mortality. 
The overall mortality rate from $\mathrm{HLH}$ ranges across studies from 22 to $59 \%[12]$. In recent years, a trend to a reduction in mortality among critically ill patients with SHLH admitted to the ICU was observed. In the sub-group of pediatric patients, the same trend was also found. In 2018, Gregory and colleagues described a single center experience of HLH in a PICU over a 10-year period, which including 42 patients, overall initial PICU hospitalization mortality and 1 -year mortality was $21 \%$ and $42 \%$ respectively[13]. This outcome benefits may be owed to improvement in chemotherapy, multiple organ support technology and better intensive care management. Nevertheless, there is no published literature reporting mortality in SHLH-associated MODS patients required CRRT. In our registry, total initial PICU hospitalization mortality for our patient cohort was $46.2 \%(22 / 52)$. This may shed light onto the quite poor outcomes among patients with SHLH and MODS who need CRRT. Thus, the development of therapies targeted at preventing or limiting the progress of MODS is urgently needed.

MODS is the leading cause of hospital death in SHLH patients and the prognosis depends mainly on the number and severity of organ dysfunctions. Numerous studies reported over half of deaths occur within 30 days after SHLH diagnosis due to MODS or second infection[14, 15]. Leow et al[16], assessed poor prognostic factors and mortality for pediatric patients with $\mathrm{HLH}$ admitted to the cardiac ICU, they found patients with a higher mortality index score at admission, higher serum lactate levels, the need for mechanical ventilation, vasoactives and CRRT were associated with higher mortality. Indeed, we demonstrated a correlation between the number of organ dysfunctions and mortality. Significantly, nonsurvivors had more respiratory, cardiovascular and gastrointestinal dysfunctions. Likewise, the use of organ support (mechanical ventilation, vasoactive agents) was also higher in the nonsurvivors.

Abrupt onset of rapid progress to MODS is common in fatal HLH patients[17] MODS is often reported at the advanced stage of SHLH and is related to abnormally higher concentrations of hypercytokinemia including interferon- $\gamma($ IFN- $\gamma$ ), tumoral necrosis factor- $a$ (TNF- $a$ ), interleukin-10 (IL- 10), IL-6 and so on[18]. IL-6 amplifies TLR-induced inflammatory response also in cells originating from inflammatory site. In vitro, Claudia et al. demonstrated that, prolonged exposure of human macrophages to IL-6 leads to increased production of cytokines, including (C-X-C Motif) Ligand 8 (CXCL-8) and Tumor Necrosis Factor$a(T N F-a)[19]$. A single-center study reported, renal failure was related to abnormally high concentrations of nephrotoxic interleukin-6 (IL-6) in serum[20]. Our data demonstrated that serum IL-6 levels was positively associated with the number of dysfunction organs on correlational analysis which corroborated similar findings from previous studies.

The prognostic significance of cytokines for early death has once been reported[21]. Tang et al[22] found high IFN-y and IL-10 levels were associated with early death. It is worth noting that, in our study serum IL6 levels at PICU admission was higher in the non-survivor group than survivor group. We further found that highly elevated IL-6 level (>13.12 pg/mL) was an independent risk factor of hospital death in critically SHLH-associated MODS pediatric patients. This result was consistent with previous study of $\mathrm{HLH}$, which showed patients in the non-survivors group had higher IL-6, IFN- $y$ and IL-10 levels[23]. IL-6 levels are usually significantly elevated in patients with sepsis, which is considered to be the major cause of mobility and mortality in pediatric SHLH patients[24]. This may partially explain the phenomenon of 
higher level of IL-6 in SHLH patients with death. As a result, it may be reasonable to consider IL-6 levels greater than $13.12 \mathrm{pg} / \mathrm{ml}$ as a prognostic factor maker of SHLH.

Current management of SHLH-associated MODS includes prompt clinical stabilization with ICU-level organ supportive care, applying specific measures to control the hyperinflammation, identification and treatment of the underlying cause of SHLH and infectious complications. From the onset, aggressive interventions for the treatment of multiorgan dysfunction are usually conducted to stabilize the patient's status while allowing time for other therapeutic strategy to treat SHLH. Management of the hyperinflammatory response focuses on blocking excessive cytokine production and eliminating the triggers are also important[14,25]. Medications, such as corticosteroids and immunosuppressants, are recommended in SHLH treatment to suppress the inflammatory response and control cell proliferation[9, 26]. However, corticosteroids and immunosuppression leave many SHLH patients susceptible to infection, as well as secondary infections can trigger reactivation of the underlying hyperinflammatory response leading to additional morbidity and mortality.

CRRT as a new type of renal replacement, is being used more often in critically ill children with MODS. CRRT has been also recommended as an effective therapeutic for the treatment of systemic inflammatory syndromes. With regard to severe sepsis adjunctive therapies, inflammatory mediator modulation can be achieved through hemofiltration (HF) based CRRT mode, such as continuous venovenous hemofiltration (CVVH) or continuous venovenous hemodiafiltration (CVVHDF). One of the mechanisms for the beneficial effect of HF in sepsis may be the convective clearance of soluble inflammatory mediators. Over the last few years, animal experiments and human studies have shown that, $\mathrm{CVVH} / \mathrm{CVVHDF}$ can remove soluble inflammatory mediators of sepsis, as well as attenuate the severity of the response to sepsis. DiCarlo et al[27] demonstrated the utility of continuous hemofiltration in attenuating the consequences of excess cytokine activity and the degree of lactate in three HLH patients with MODS. Our previous single-center nonrandomized concurrent control trial showed highvolume hemofiltration (HVHF) may be an effective adjunctive treatment in SHLH/MAS by improving organ function and decreasing serum level of TNF- $a$ and IL-6[8]. There are several small reports of treatment of SHLH with alternative therapeutics directed against IL-1, IL-6 and TNF-a in serum, which have demonstrated clinical benefits for these patients[28].

Our study has some limitations. First, gene sequencing was not performed in all patients, so that the proportion of FHL remained uncertain. Second, although this study was based on a multi-centered prospective study, relatively small number of patients was warranted to validate the results.

\section{Conclusion}

In summary, highly number of organ dysfunction ( $\geq 3)$, and highly increased IL-6 ( $\geq 13.2 \mathrm{pg} / \mathrm{mL})$ at CRRT initiation are independent prognostic factors for predicting early death in children with SHLH and MODS. These findings may help guide the treatment decision making for this disease to avoid insufficient 
therapy. In addition, IL-6 can be designated as a particular cytokine biomarker for SHLH- associated MODS patients. Inhibiting IL-6 may be a potential therapeutic strategy for reversing these cases.

\section{Abbreviations}

MODS

Multiple organ dysfunction syndrome

PICU

pediatric intensive care unit

ICU

intensive care unit

SHLH

secondary hemophagocytic lymphohistiocytosis

CRRT

continuous renal replacement therapy

IAHS

infection-associated hemophagocytic

MAS

macrophage activation syndrome

M-HLH

malignancy-associated hemophagocytic lymphohistiocytosis

CRF

case report forms

$\mathrm{CVVH}$

continuous venovenous hemodialysis

CVVHDF

continuous venovenous hemodiafiltration

CRRT

continuous renal replacement therapy

APTT

activated partial thromboplastin time

RCA

regional citrate anticoagulation

PRISM III

pediatric risk of mortality III

IQR

interquartile range

ROC

receiver operating characteristic

FO 
fluid overload

HVHF

high-volume hemofiltration

$\mathrm{HF}$

hemofiltration

TBIL

total bilirubin

DBIL

direct bilirubin

ALB

albumin

PLT

platelet counts

PT

prothrombin time

FIB

fibrinogen

BUN

serum urea nitrogen

$\mathrm{CR}$

serum creatinine

$\mathrm{HB}$

hemoglobin

NK

natural kill cell

OR

odd ratio

AKI

acute kidney injury

IL-1

interlukin-1

IL-6

interlukin-6

TNF-a

tumor necrosis factor-a

IFN-Y

interferon- $\gamma$

IL-10

interlukin-10 


\section{Declarations}

\section{- Ethics approval and consent to participate}

Not applicable.

\section{- Consent for publication}

Not applicable.

\section{- Availability of data and materials}

The datasets generated and/or analysed during the current study are not publicly available due to the datasets are now having secondary analysis but are available from the corresponding author on reasonable request.

\section{- Competing interests}

The authors declared no potential conflicts of interest with respect to the research, authorship, and/or publication of this article.

\section{- Funding}

This study was supported by New Advanced Technology Project at the Shanghai City Hospital Development Center (SHDC12014116), Modern Western Medicine Guiding Project of Shanghai Science and Technology Committee (18411951000, 17411968900), Key developing Subject Program from Shanghai Municipal Commission of Health and Family Planning (No.2016ZB0102).

\section{- Authors' contributions}

YCZ and YC designed the study. JYS, YJS, YNZ, GFY and HR managed data and its quality. JYS, YJS and CXW performed the statistical analysis. YC and JYS drafted the manuscript. CXW and YCZ contributed substantially to its revision. All authors read the manuscript carefully and approved the final version.

\section{- Acknowledgements}

Not applicable.

\section{References}

1. Tothova Z, Berliner N. Hemophagocytic syndrome and critical illness: New insights into diagnosis and management. J Intensive Care Med. 2015, 30:401-412.

2. Apodaca E, Rodríguez-Rodríguez S, Tuna-Aguilar EJ, Demichelis-Gómez R. Prognostic Factors and Outcomes in Adults With Secondary Hemophagocytic Lymphohistiocytosis: A Single-center 
Experience. Clin Lymphoma Myeloma Leuk. 2018;18: e373-e380.

3. Créput C, Galicier L, Buyse S, Azoulay E. Understanding organ dysfunction in hemophagocytic lymphohistiocytosis. Intensive Care Med. 2008; 34:1177-187.

4. Gregory J, Greenberg J, Basu S. Outcomes Analysis of Children Diagnosed With Hemophagocytic Lymphohistiocytosis in the PICU. Pediatr Crit Care Med. 2019;20: e185-e190.

5. Karapinar B, Yilmaz D, Balkan C, Akin M, Ay Y, Kvakli K. An unusual cause of multiple organ dysfunction syndrome in the pediatric intensive care unit: hemophagocytic lymphohistiocytosis. Pediatr Crit Care Med. 2009;10: 285-290.

6. Ronco C, Ricci Z, De Backer D, Kellum JA, Taccone FS, Joannidis M, et al. Renal replacement therapy in acute kidney injury: controversy and consensus. Crit Care. 2015;19:146.

7. Wu C, Wang X, Yu W, Li P, Liu S, Li J, et al. Short-term consequences of continuous renal replacement therapy on body composition and metabolic status in sepsis. Asia Pacific journal of clinical nutrition. 2016,25(2):300-7.

8. Cui Y, Zhang Y C, Kang Y L, Ren YQ, Miao HJ, Wang F. High-Volume Hemofiltration in Critically III Patients With Secondary Hemophagocytic Lymphohistiocytosis/Macrophage Activation Syndrome: A Prospective Study in the PICU. Pediatr Crit Care Med. 2016;17:e437-e443.

9. Henter JI, Horne A, Aricó M, Egeler RM, Filipovich AH, Imashuku S, et al. HLH-2004: Diagnostic and therapeutic guidelines for hemophagocytic lymphohistiocytosis. Pediatr Blood Cancer. 2007, 48(2): 124-131.

10. Goldstein B, Giroir B, Randolph A, International Consensus Conference on Pediatric Sepsis. International Consensus Conference on Pediatric Sepsis International pediatric sepsis consensus conference: definitions for sepsis and organ dysfunction in pediatrics. Pediatr Crit Care Med. 2005, 6:2-8.

11. Youden WJ. Index for rating diagnostic tests. All statistical analyses were performed using SPSS (version 19, IBM-SPSS, SPSS Inc. New York, USA software. Cancer. 1950;3:32-35.

12. Al-Samkari H. Hemophagocytic Lymphohistiocytosis. Annu Rev Pathol. 2018;13:27-49.

13. Gregory J, Greenberg J. Outcomes Analysis of Children Diagnosed With Hemophagocytic Lymphohistiocytosis in the PICU. Pediatr Crit Care Med. 2019;20: e185-e190.

14. Bin Q, Gao JH. Prognostic factors of early outcome in pediatric hemophagocytic lymphohistiocytosis: an analysis of 116 cases. Ann Hematol. 2016;95:1411-1418..

15. Arca, L. Fardet L. Galicier L, Rivière S, Marzac C, Aumont C, et al. Prognostic factors of early death in a cohort of 162 adult haemophagocytic syndrome: impact of triggering disease and early treatment with etoposide. Br J Haematol. 2015, 168 (1): 63-68.

16. Leow EH, Soh SY, Tan AM, Mok YH, Chan MY, Lee JH. Critically ill children with hemophagocytic lymphohistiocytosis: A case series of 14 patients. J Pediatr Hematol Oncol. 2017, 39:e303-e306.

17. Nahum E, Ben-Ari J, Stain J, Schonfeld T. Hemophagocytic lymphohistiocytic syndrome: Unrecognized cause of multiple organ failure. Pediatr Crit Care Med. 2000, 1:51-54. 
18. Jordan MB, Hildeman D, Kappler J, Marrack P. An animal model of hemophagocytic lymphohistiocytosis (HLH): CD8+ T cells and interferon gamma are essential for the disorder. Blood. 2004,104:735-43.

19. Caiello I, Minnone G, Holzinger D, VogI T, Prencipe G, Manzo A, et al. IL-6 amplifies TLR mediated cytokine and chemokine production: implications for the pathogenesis of rheumatic inflammatory diseases. PLoS One. 2014; 9(10):e107886.

20. Aulagnon F, Lapidus N, Canet E, Galicier L, Boutboul D, Peraldi MN, et al. Acute kidney injury in adults with hemophagocytic lymphohistiocytosis. Am J Kidney Dis. 2015, 65(6):851-9.

21. Brisse $E$, Wouters $\mathrm{CH}$, Matthys P. Hemophagocytic lymphohistiocytosis $(\mathrm{HLH})$ : A heterogeneous spectrum of cytokine-driven immune disorders. Cytokine Growth Factor Rev. 2015, 26(3):263-80.

22. Yang SL, Xu XJ, Tang YM, Song H, Xu WQ, Zhao FY, et al. Associations between inflammatory cytokines and organ damage in pediatric patients with hemophagocytic lymphohistiocytosis. Cytokine. 2016,85:14-17.

23. Tang YM. Advances in hemophagocytic lymphohistiocytosis: pathogenesis, early diagnosis/differential diagnosis, and treatment. Scientific World Journal. 2011;11:697-708.

24. Tang Y, Xu X, Song H, Yang S, Shi S, Wei J, et al. Early diagnostic and prognostic significance of a specific Th1/Th2 cytokine pattern in children with haemophagocytic syndrome. British journal of haematology. 2008, 143(1):84-91.

25. Chandrakasan S, Filipovich AH. Hemophagocytic Lymphohistiocytosis: Advances in Pathophysiology, Diagnosis, and Treatment. The Journal of pediatrics. 2013, 163(5):1253-1259.

26. Bode SF, Lehmberg K, Maul-Pavicic A, Gera R. Recent advances in the diagnosis and treatment of hemophagocytic lymphohistiocytosis. Arthritis Res Ther. 2012;14:213.

27. DiCarlo J, Lui WY, Frankel L, Howell W, Schiffman J, Alexander S. The hemophagocytic syndrome: Titrating continuous hemofiltration to the degree of lactic acidosis. Pediatr Hematol Oncol. 2006, 23:599-610.

28. Mostaza-Fernández JL, Guerra Laso J, Carriedo Ule D, Ruiz de Morales JM. Hemophagocytic lymphohistiocytosis associated with viral infections: Diagnostic challenges and therapeutic dilemmas. Rev Clin Esp. 2014;214:320-327.

\section{Figures}




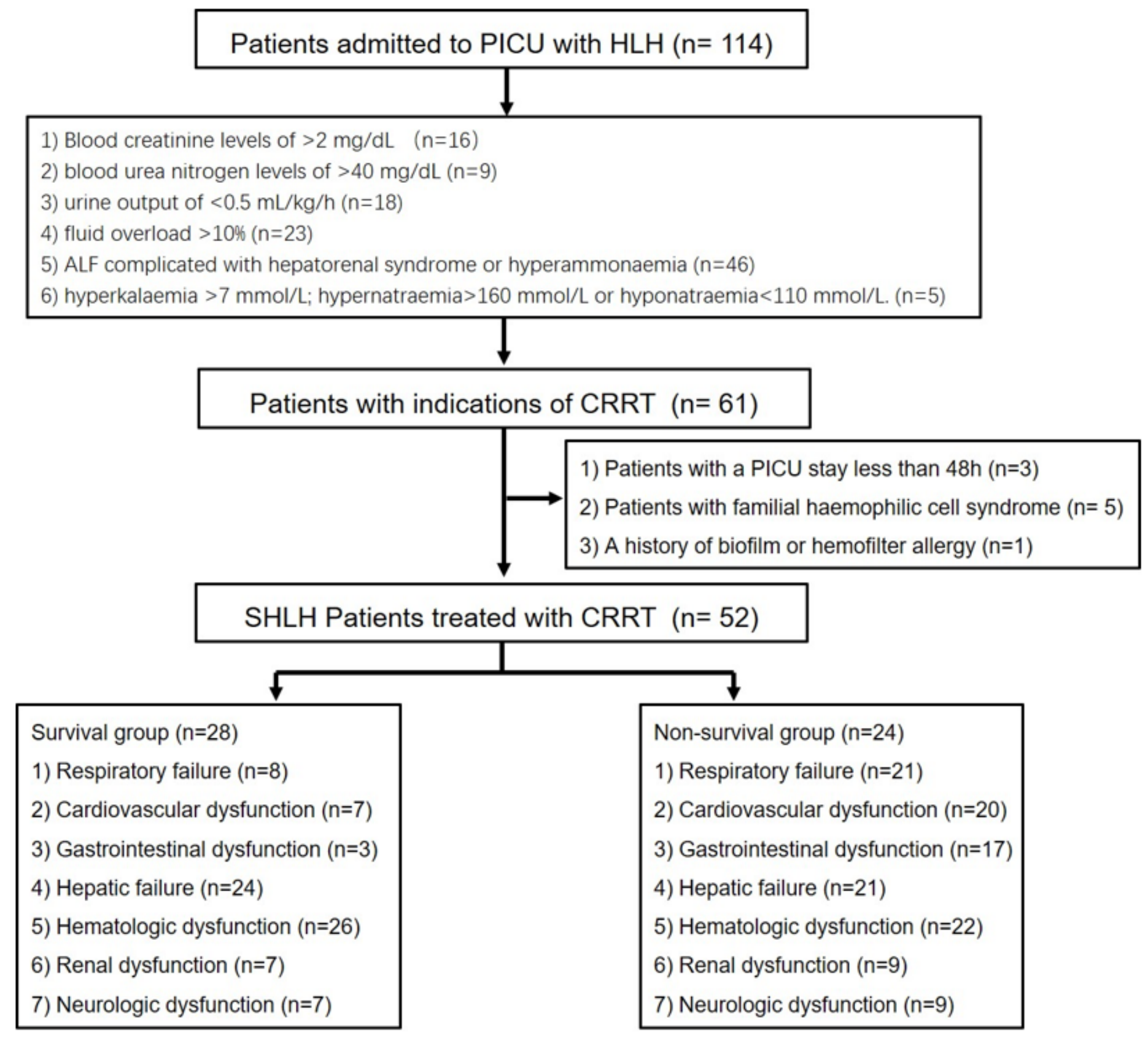

Figure 1

Flowchart of patients with SHLH enrolled in this multicenter study. 


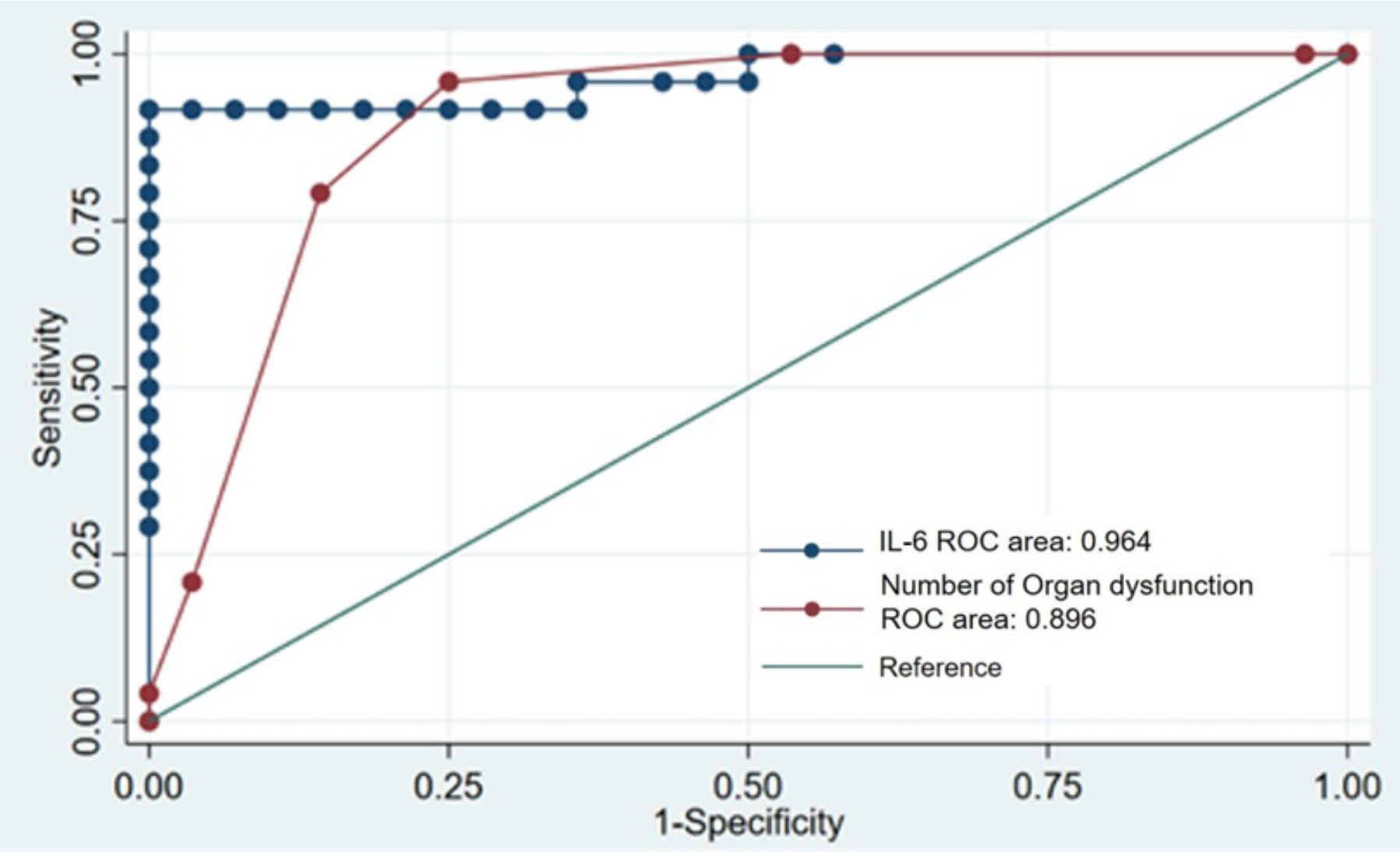

Figure 2

ROC analysis of IL- 6 and number of organ dysfunction in the PICU mortality of patients with SHLH and MODS

\section{Supplementary Files}

This is a list of supplementary files associated with this preprint. Click to download.

- STROBEchecklistSHLH0206.docx 